\title{
ENSEÑANZA DEL ESPAÑOL COMO LENGUA EXTRANJERA PARA NIÑOS: CLASES MÁS INTERACTIVAS
}

\author{
Dércia Thays Araújo Soares ${ }^{1}$
}

RESUMEN: Los niños tienen facilidad para aprender, esto es un hecho. La enseñanza de español en la educación infantil ya viene conquistando un espacio. Las estrategias lúdicas son muy adecuadas para esta etapa de la enseñanza. Pero lo lúdico no quiere decir "fijo" tampoco "improviso". Es necesario que el profesor de la enseñanza infantil se mantenga al día con las nuevas tecnologías, estrategias y materiales didácticos ofrecidos por las investigaciones lingüísticas. Teniendo en cuenta que los profes de la enseñanza infantil suelen seguir un material didáctico fijo, designado por la escuela, proponemos clases flexibles de base en la Lingüística Aplicada. Basado en los estudios de Elena Benitiz Rodriguez (2020) y anclado en las investigaciones de Ausubel (1968). Propondremos 3 clases usando estrategias de diferentes campos lingüísticos, bajo las investigaciones propias de la Lingüística aplicada.

Palabras-claves: Enseñanza. E/LE. Niños. Métodos.

RESUMO: As crianças têm facilidade para aprender, isso é um fato. $O$ ensino de espanhol na educação infantil vem conquistando um espaço. As estratégias lúdicas são muito apropriadas para esta fase do ensino. Mas o lúdico não significa "fixo" ou "improvisado". É necessário que os professores do ensino infantil se mantenham atualizados em relação às novas tecnologias, estratégias e materiais didáticos oferecidos por pesquisas linguísticas. Tendo em conta que os professores do ensino infantil seguem um material didático fixo, designado pela escola, propomos classes flexíveis com base na Linguística Aplicada. Baseado nos estudos de Elena Benitiz Rodriguez (2020) e ancorado nas investigações de Ausubel (1968). Propormos 3 aulas usando estratégias e diferentes campos linguísticos, sob a investigação da lingüística aplicada.

Palabras-chave: Ensino. E/LE. Crianças. Métodos.

\section{INTRODUCCIÓN}

La educación infantil es considerada una de las etapas más importantes para la formación de los niños. Es la primera etapa de la educación básica, que atiende a niños de cero a cinco años, y es cuando empiezan a vivir fuera del entorno familiar.

La educación infantil se define en la Ley de Directrices y Bases de la Educación Nacional (LDB) como parte de la educación básica, pero no de la educación obligatoria, sus conocimientos fueron adquiridos desde 1974, cuando el tema fue discutido por algunos consejeros en el consejo federal de educación y

\footnotetext{
${ }^{\mathrm{I}}$ Discente do curso de Letras - Língua Espanhola e Literaturas - Licenciatura pela Universidade Federal do Rio Grande do Norte. Técnica em Telecomunicações pelo SENAI - Serviço Nacional de Aprendizagem Industrial. EMAIL: derciathays@hotmail.com
} 
conquistando espacio a nivel nacional con la nueva LDB, ley de de directrices y bases (ley n 9394/96), que trajo más atención a los niños menores de 6 años. (POLO e PEDRAÇA, p. or)

Es un ambiente donde hay un mayor desarrollo del niño, porque la inteligencia se construye a partir del entorno físico y social en que vive el niño. Esta etapa es importante, el niño tendrá una base para cuando entre en la escuela primaria, donde empezará a desarrollar la alfabetización, el alumno que se salte esta fase se quedará atrás con respecto a los demás alumnos, ya que las habilidades se construyen durante esta fase.

Los niños están en una etapa en que la curiosidad es enorme, ellos están encantados con todo lo que es nuevo, especialmente cuando se trata de una lengua extranjera. $Y$ en esta etapa, el aprendizaje de un nuevo idioma se torna más fácil. Así, los profesores necesitan una búsqueda constante de aprendizaje y desarrollo en sus competencias, no pueden limitarse a enseñar con los libros didácticos, tiene que buscar clases donde los alumnos tengan interés y ganas de participar.

En esta edad la enseñanza de la lengua española puede ser a través de juegos, actividades que atraigan su atención y que les hagan desarrollar su imaginación, es en este periodo cuando ellos buscan la interacción, la diversión, y por eso la forma de trabajar con los niños, es muy importante.

Teniendo en cuenta esta información, el objetivo de este artículo es que los profesores de español de las escuelas infantiles reflexionen sobre el método que están utilizando en sus clases y cómo pueden aplicar una forma de enseñanza más interactiva.

Para eso, nos basamos en los estudios de Elena Benitiz Rodríguez (2020) y anclados en las investigaciones de Ausubel (1968), que contribuyeron a nuestros estudios. Con ello, esta investigación pretende aportar tres ejemplos de lecciones interactivas que sirvan de ejemplo y apoyo a los profesores de educación infantil.

\section{MARCO TEÓRICO}

La Lingüística Aplicada es un campo de estudio que investiga y busca soluciones para problemas relacionados a la lengua, es una parte de la lingüística que propone la aplicación de las teorías y conocimientos propios a la resolución de problemas diversos. En otras palabras, ella se interesa por aplicaciones de la lingüística en otras áreas de la vida. Pero no se trata de cualquier problema, y si de problemas que tiene relevancia social que exigen respuestas teóricas y que traiga beneficios a la práctica social, también tiene un 

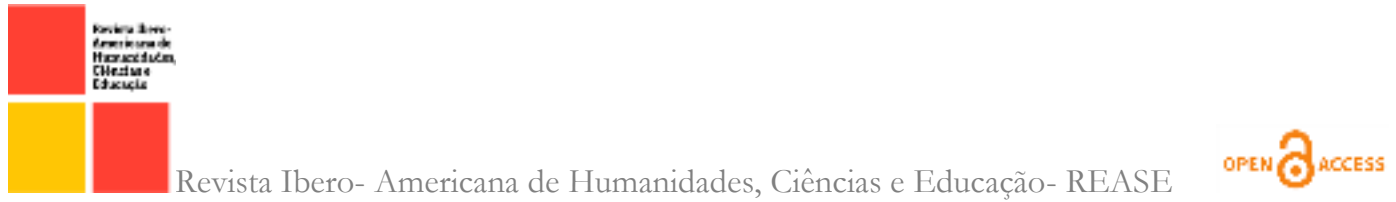

diálogo con otras áreas de estudios, por ejemplo, la educación, la filosofía, la psicología, antropología, política, sociología y otras.

En la enseñanza del español como lengua extranjera en la educación infantil, es posible aplicar la Lingüística Aplicada, trayendo sus teorías para basarse en los planes de clase, haciendo con que las clases sean más interactivas. Considerando que la labor de la Lingüística Aplicada es proponer salidas interactivas a la enseñanza de idiomas, principalmente, podemos involucrar la lingüística aplicada en las clases de español, aportando lecciones interactivas y buscando temas de nuestro cotidiano. David Ausubel, psicólogo educativo, que tiene la teoría del aprendizaje, habla sobre la aprendizaje significativo, que se refiere a un proceso de una nueva información que se relaciona con un aspecto relevante de la estructura de conocimiento del individuo.

La Lingüística Aplicada se basa justamente en esto, las clases de ELE pueden ser interactivas, no solamente en la enseñanza infantil, sino en todos los niveles. Son clases en las que se puede tener el cotidiano y también se utilizan las teorías como base para aquella determinada actividad. Y podemos observar que hay una diferencia en las clases que el profesor sigue todo lo que el libro determina y una clase que tiene el calentamiento, la explicación, la práctica controlada y una libre.

Esta singularidad supone, por un lado, asumir que enseñar una lengua extranjera a adultos no es igual que enseñarsela a los niños, pues estos son más intuitivas, no la comparan con su primera lengua, ofrecen generalmente menos resistencia a aprender estructuras nuevas y piensan más en el significado de la comunicación que en la forma del mensaje, por lo que su aprendizaje es más cercano al proceso natural de la adquisición. (BENITEZ, 2020, p. 2)

Los niños aprenden con facilidad, por medio de la interacción de los relacionamentos significativos, es cuando ellas establecen un vínculo con otro niño, con un adulto. No tienen miedo de cometer errores, no están tan preocupadas con lo que los otros van a decir, y están en una fase muy buena, que es la fase donde aprende con los adultos. Pero para que los niños tengan interés en aprender lo que el profesor quiere que aprendan, las clases tienen que ser interactivas. Cuanto más interactivas más aprenden, porque están en la etapa de búsqueda de la infancia.

Benitez (2020) escribe sobre cómo puede ser la formación de un profesor para dar clases a niños, los profesores tienen siempre buscar nuevas formas de aplicar la lengua extranjera en las clases y esta ayuda podemos buscar en la Lingüística Aplicada. Las clases 


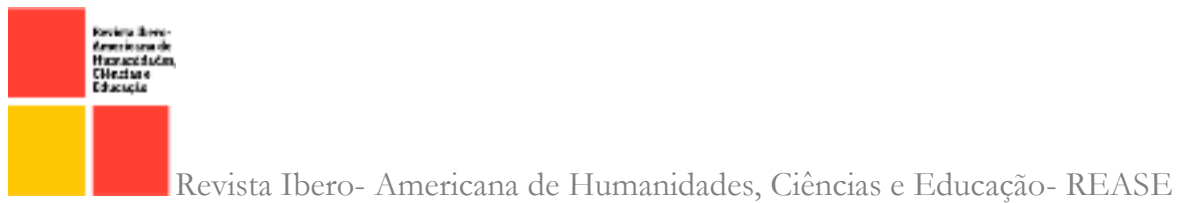

interactivas son la mejor forma de dar clases para niños, en la educación infantil. Piaget es un estudioso que escribe sobre esto.

Según la visión de Piaget, Vigotsky considera que el aprendizaje de un niño es más significativo y duradero cuando se les enseña mediante actividades lúdicas por medio de las cuales ellos tengan la oportunidad de explorar y descubrir el mundo que les rodea a través de su interacción con él. (BARAJAS, RUBIO, VALDIVIA, p. r)

Según Benitez (2020) Las clases de ELE de la educación infantil pueden ser aplicadas con nuevas ideas innovadoras, con canciones, cuentos, juegos que hagan que los niños aprendan y se diviertan.

Los niños tienen una gran facilidad para aprender cosas nuevas y enseñarles un idioma es bueno. Para que se interesen en conocer y aprender cosas nuevas, las lecciones tienen que ser interesantes y con ideas que llamen su atención. La interacción con otros niños, otras personas, también es un punto importante en el aprendizaje de ellos. Los niños aprenden en los más diversos contextos de espacios, no solamente en la escuela, más en casa, no parque, con sus familiares, o sea, en todo lugar.

\section{ENSEÑANZA NIÑOS Y ADULTOS: DIFERENCIAS}

Sabemos que los niños y los adultos aprenden de manera diferente, los niños aprenden de una manera y los adultos de otra, y cómo se aplicará esto en las lecciones de ELE es responsabilidad e interés de los educadores. Los profesores deben comprender la diferencia entre los dos grupos y basarse en ella para crear clases. Cada grupo necesita una enseñanza diferente para tener un aprendizaje de calidad.

Los niños están con la mente hecha para aprender, ellos captan con facilidad cualquier tipo de estímulo, y no tiene miedo de errar. Un adulto no aprende al mismo ritmo que un niño, su cerebro está acostumbrado a reforzar el aprendizaje.

Según Fredrick (2018) la enseñanza de uno para otro es muy distinta y podemos destacar algunos puntos que diferencia el aprendizaje de ellos. La primera es la dirección:

Los niños necesitan orientación en el proceso de aprendizaje. Para la mayoría de los niños, la falta de estructura y orientación en el estudio genera caos, falta de aprendizaje del material requerido. Los adultos trabajan con un método diferente. Están acostumbrados a ser autodirigidos y necesitan ser estimulados para guiarse a sí mismo cuando se estudia. (FREDRICK, 2018)

Los alumnos de la enseñanza infantil necesitan estar siempre siendo guiados por su profesor para tener una buena aprendizaje, es la fase que ellos tiene el maestro como un ejemplo, entonces es más fácil hacer con que ellos aprendan. Ya el adulto no funciona de 
la misma forma, los adultos tienen una "cabeza" lista y tiene que ser enseñado con un método distinto.

El segundo punto que Fredrick (2018) dice, es propósito:

\begin{abstract}
A los niños les resulta fácil aprender las cosas de memoria. No tienen una vida de experiencia para dar contexto a su educación, por lo que a menudo la manera más fácil de enseñar a un niño es simplemente presentar la información de varias maneras diferentes hasta que la memorizan. Esto es más difícil con los adultos. Ellos tienden a requerir un propósito o dirección en sus estudios con el fin procesar la nueva información dentro de su marco de referencia existente. (FREDRICK, 2018)
\end{abstract}

La memoria de un niño es más fresca, totalmente diferente de la memoria de una persona adulta, que está llena de ocupaciones y necesitan de un apoyo para sus estudios. Para el niño, repetir aquella misma cosa algunas veces, ya hace que ellos memoricen y es muy difícil de olvidar.

El tercer punto de Fredrick (2018), es conocimiento existente:

Los profesores tienen que entender lo que los estudiantes adultos saben sobre un tema determinado, con el fin de enseñar con eficacia a una clase llena de adultos. Cuando se enseña a los jóvenes, es fácil suponer o deducir lo que los estudiantes saben. (FREDRICK, 2018)

En una clase de niños, los profesores saben lo que ellos necesitan saber en cada edad, es más fácil traer un asunto para ellos. Ya en una clase de adultos, no es tan fácil, necesitamos traer un asunto con todas las informaciones, pues algunos pueden tener un gran conocimiento sobre aquel terminado contenido y otros no. Entonces debemos tener todo cuidado con esto.

El último punto que el habla es el tiempo:

\begin{abstract}
Los niños en la clase no están preocupados por el tiempo, excepto en la medida en que quieren que la clase se acabe para que puedan salir a jugar. Sin embargo, el valor real del tiempo no es algo que se hace evidente hasta la edad adulta. Los adultos valoran su tiempo grandemente y tienen que sopesar el tiempo dedicado al aprendizaje contra otras responsabilidades. El conocimiento directo, aplicable es de mayor interés para los adultos, ya que por lo general no quieren perder tiempo en temas amplios o generalidades. (FREDRICK, 2018)
\end{abstract}

Cuando un adulto decide aprender un nuevo idioma, ellos intentan ganar todo el tiempo posible para tener éxito, en las clases van a preguntar todo que tiene curiosidad y siempre intentan hablar más rápido. Para los niños, esto es una diversión y con clases interactivas entonces, ellos no tienen con qué preocuparse, solamente con las brincadas con sus colegas o en la hora de ir para su casa.

Esto fue solamente algunas diferencias que tiene entre la enseñanza de lengua española para niños y la enseñanza para adultos, pero podemos encontrar más. Es bueno 
que los niños aprendan un nuevo idioma cuando están en esta fase, pues están con la mente fresca y aprende todo lo que el profesor les dice, principalmente aquellos niños que pegan su profe como ejemplo.

\section{CLASES PROPUESTAS}

Según Dolz (2004), Una "secuencia didáctica" es un conjunto de actividades escolares organizadas sistemáticamente en torno a un género textual oral o escrito. La secuencia didáctica es un módulo de presentar un contenido en varias partes con profundidad, es tomar un asunto y dividir, haciendo diversas tareas para la mejor comprensión del alumno. Y vamos a proponer una secuencia para enseñar a los niños de forma interactiva.

\section{-PLAN i LOS ANIMALES}

El objetivo de esta clase es que los alumnos sean capaces de identificar los animales.

Actividad inicial: Pondremos la canción de los animales, como introducción al tema, tres veces, para que puedan bailarla y escucharla.

https://www.youtube.com/watch?v=RDVzPniYWWc

Duración de la actividad: ro minutos.

Actividad principal: Les enseñaremos dos veces cuatro cartón con vocabulario de los animales que aparece en la canción y que en la medida de lo posible se les puede enseñar mediante las figuras del arca con los animales. Deberán repetir cómo se pronuncia. Después cada niño dibujará una de las fotos.

Duración de la actividad: Io minutos.

Actividad final: Para finalizar, se les contará el cuento El carnaval de los animales, con marionetas.

https://www.youtube.com/watch?v=2k2IZceeytQ

Duración de la actividad: 20 minutos.

\section{-PLAN 2 COMO SON}

El objetivo de esta clase es que los alumnos sean capaces de describir los animales.

Actividad inicial: Volveremos a poner la canción de los animales. 
https://www.youtube.com/watch?v=RDVzPniYWWc

Duración de la actividad: Io minutos.

Actividad principal: Observaremos los diferentes animales que aparecen en el vídeo proyectado de la canción.

Duración de la actividad: 20 minutos

Actividad final: Juego de equipo en que ellos van a encontrar por la clase animales de peluche que hayan aparecido en la canción.

Duración de la actividad: ro minutos.

\section{-PLAN 3 QUIÉN ES QUIÉN}

Con las siguientes actividades se va a poder diferenciar los géneros de los animales. Actividad inicial: Comenzaremos introduciendo la clase bailando y cantando la canción de las clases anteriores.

Duración de la actividad: 5 minutos.

Actividad principal: Se les mostrarán los animales en peluche asociando cada uno con su nombre escrito (apoyo visual). Después, todos juntos intentaremos recordar el nombre, de manera oral, tras varias repeticiones, de cada animal.

Duración de la actividad: is minutos

Actividad final: Veremos la diferencia que hay entre masculino y femenino (el/la), de manera lúdica, buscando objetos variados por toda la clase, de manera que el profesor sea quien le ponga el determinante delante, para indicar si es masculino o femenino.

Duración de la actividad: 20 minutos.

\section{CONCLUSION}

A través de este artículo intentamos presentar cómo podemos enseñar a los niños de forma interactiva con el apoyo de la Lingüística Aplicada, y cómo podemos llamar su atención para que sean capaces de aprender un nuevo idioma de una forma divertida. Como hablamos, la educación infantil es considerada una de las etapas más importantes para la formación de los niños. Es una buena hora para aprender dos idiomas, pues ellos aprenden con facilidad y cuando las clases son buenas, con cosas que les gustan, es mejor. 
También mostramos que en esta etapa, los niños están en la fase que son más curiosos, se quedan encantados con todo que es nuevo, especialmente cuando se trata de una lengua extranjera. Y con la enseñanza interactiva, ellos aprenden más rápido, con facilidad y con el apoyo de la Lingüística Aplicada, podemos obtener estos resultados.

Para que esto ocurra, los profesores deben estar siempre actualizando y buscando nuevas cosas para tornar sus clases atractivas e interactivas, no se puede perder tiempo, tiene que buscar y buscar. Y para finalizar, también colocamos una secuencia didáctica que sirve como ejemplo de clases interactivas, que tiene una ligación una con otra.

\section{REFERÊNCIAS}

DOLZ, Joaquim; NOVERRAZ, Michele; SCHNEUWLY, Bernard. Sequências didáticas para o oral e a escrita: apresentação de um procedimento. In: SCHNEUWLY, Bernard; DOLZ, Joaquim. Gêneros orais e escritos na escola. Campinas: Mercado de Letras, 2004. p. $8 \mathrm{I}-\mathrm{I}$ - 8.

FREDRICK, Hans. Diferencias entre el aprendizaje infantil y el adulto. eHow en Español, I5 de mayo, de 2018. Disponible en: <https://www.ehowenespanol.com/diferencias-entre-el-aprendizaje-infantil-y-eladulto_13104979/> Accedido en: 30 de agosto de 2021.

FERNANDES, Elisângela. David Ausubel e a aprendizagem significativa. Nova Escola, or de diciembre de 20Ir. Disponible en: <https://novaescola.org.br/conteudo/262/davidausubel-e-a-aprendizagem-significativa\#> Accedido en: 25 de agosto de 2021.

MEDEIRO, M. H.; LEAL, O. B. S.; SILVA, R. C. A.; GOUVEIA, A. B. O Ensino De Espanhol Como Língua Estrangeira Para Crianças, Paraíba.

POLO, A. T. A Importância Da Educação Infantil Para O Desenvolvimento Pleno Da Criança. Conic Semes. Disponible en: <http://conicsemesp.org.br/anais/files/2015/trabalho-ıooo2IIo5.pdf> Accedido en: 26 de agosto de 2021.

RODRÍGUEZ, E. B. La enseñanza del español como lengua extranjera a niños: una propuesta didáctica para Educación Infantil. Revista Electrónica del Lenguaje, no 7, 2020, Canadá, 02 de Noviembre de 2020. 